\title{
Consumption of an aqueous cyanophyta extract derived from Arthrospira platensis is associated with reduction of chronic pain: results from two human clinical pilot studies
}

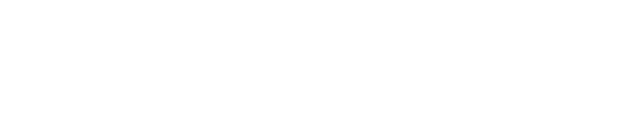

Gitte S Jensen'

Victoria L Attridge'

Steve G Carter'

Jesse Guthrie ${ }^{2}$

Axel Ehmann²

Kathleen F Benson'

'NIS Labs, ${ }^{2}$ Cerule LLC, Klamath Falls, OR, USA
Correspondence: Gitte S Jensen NIS Labs, I 437 Esplanade, Klamath Falls, OR 9760I, USA

Tel + I $54 \mid 8840112$

Fax +I 403 44I 5236

Email gitte@nislabs.com
Objectives: The aim of this study was to evaluate the effects of consumption of an aqueous cyanophyta extract (ACE) from Arthrospira platensis on chronic pain in humans, in two clinical pilot studies.

Design and interventions: The two pilot studies each involved 12 subjects experiencing chronic pain. The initial study followed an open-label 4-week study design involving consumption of $1 \mathrm{~g}$ ACE per day. A subsequent placebo-controlled, single-blind, crossover study involved consumption of $500 \mathrm{mg} \mathrm{ACE}, 250 \mathrm{mg} \mathrm{ACE}$, or $0 \mathrm{mg} \mathrm{ACE}$ (placebo) per day for 1-week duration, separated by 1 -week washout period.

Subjects: Adult subjects of both sexes, with chronic joint-related pain for at least 6 months prior to enrollment, were recruited after obtaining written informed consent.

Outcome measures: Visual analog scales were used to score pain at rest and during physical activity for each person's primary and secondary areas of chronic pain. An activities of daily living questionnaire was used to collect data on physical functioning.

Results: The data showed rapid reduction of chronic pain in people consuming ACE, where the reduction in pain scores for each person's primary pain area reached a high level of statistical significance after 2 weeks of consumption $(P<0.01)$, both when at rest and when being physically active. Secondary pain areas when physically active showed highly significant improvements within 1 week of consumption of $1 \mathrm{~g} / \mathrm{d}(P<0.001)$ and borderline significant improvements within 1 week of consuming $500 \mathrm{mg} / \mathrm{d}(P<0.065)$ and $250 \mathrm{mg} / \mathrm{d}(P<0.05)$. This was accompanied by an increased ability to perform daily activities $(P<0.05)$. A small but significant weight loss was observed during the 4-week study, as the average body mass index dropped from 31.4 to $29.4(P<0.01)$.

Conclusion: Consumption of ACE was associated with reduction of chronic pain, as well as a dose-dependent increased ability to perform activities of daily living.

Keywords: activities of daily living, pilot study, placebo-controlled study, Spirulina

\section{Introduction}

Chronic joint-associated pain increases with age as a result of mechanical stress and aging and is confounded by states of chronic inflammation. The escalating need for natural methods for pain management has led to an increased interest in natural methods for reducing pain, pain perception, and inflammation. Acute pain is an essential function of our body's defense system as it stimulates motor responses to potentially dangerous stimuli. Despite pain being a sensory quality, and therefore subjective, 
it has a molecular basis including synaptic transmission and the release of excitatory neurotransmitters. ${ }^{1}$ Chronic pain, on the other hand, has no protective purpose and has been linked to the development of lowered pain thresholds due to the prolonged activation of low-threshold mechanoreceptive fibers. ${ }^{2}$ These changes in pain thresholds can result in altered pain perception and body posture compensation, which further accelerates the degradation of joint structures and reduces their functionality. ${ }^{3}$

The inflammatory cascade is a response specific to our innate immune system and involves the release of inflammatory mediators from affected cells, the production of prostaglandins via cyclooxygenase (COX-1 and COX-2) activity, and the release of cytokines and growth factors from recruited immune cells. ${ }^{4}$ Cytokines play a role in the initiation and maintenance of inflammatory states by either acting on nociceptors or indirectly stimulating the release of prostaglandins. This enhances nociceptor sensitization by reducing the activation threshold for inflammatory mediators. ${ }^{1}$ If inflammatory conditions persist, the activation of kinase cascades results in transcriptional changes that can lead to lowered response thresholds and heightened reactions to stimuli. ${ }^{1}$ In addition, sites of chronic inflammation have been linked to the origination of a variety of degenerative diseases, due to the degradation of cartilage, and linked to cancers due to DNA damage associated with persisting inflammatory and immune factors. ${ }^{5}$

In addition to their roles in the maintenance of inflammatory states, several proinflammatory cytokines have been observed to be involved in the initiation and persistence of pathological pain through their actions on nociceptive sensory neurons. ${ }^{6}$ For example, neurotrophic growth factors significantly affect neuronal sensitivity during inflammation, and increased levels of neurotrophic growth factors have been implicated in inflammatory disorders such as arthritis. ${ }^{1}$ As pain perception involves both increased production of prostaglandins by inflammatory enzymes and signal transduction pathways in the nervous system, efforts to reduce pain commonly involve both pain-reducing and anti-inflammatory measures. Currently, there is a growing interest in identifying natural products for the reduction of inflammation and the associated pain as alternatives to steroidal and nonsteroidal anti-inflammatory medications. $^{7}$ By targeting and inhibiting inflammatory pathways in a comparable way to nonsteroidal anti-inflammatory medications, natural compounds may reduce inflammatory immune responses without the side effects of prescription medications.
Antinociceptive and anti-inflammatory properties have been attributed to species of Arthrospira cyanobacteria (such as Arthrospira maxima and Arthrospira platensis), ${ }^{8,9}$ due to their naturally high concentrations of phycocyanin, phenolic compounds, and carotenoids. ${ }^{10,11}$ Lipid extracts of several species of blue-green algae were shown to decrease the nuclear translocation of nuclear factor- $\kappa \mathrm{B}$, thus contributing to decreased expression and secretion of proinflammatory cytokines. ${ }^{12}$ The pharmacological properties of phycocyanin, one of the photosynthetic components of blue-green algae, have been linked to its ability to inhibit COX-2 enzymatic activity ${ }^{13}$ and to inhibit lipid peroxidation and to scavenge free radicals in vitro. ${ }^{14}$ Phenolic compounds and carotenoids exhibit strong antioxidant properties, as they are efficient scavengers of peroxyl radicals and can donate their phenolic hydrogen atoms to neutralize free radical molecules. ${ }^{15}$ In addition, previous studies have demonstrated the clinical significance of the biological activities of Arthrospira. Specifically, Arthrospira supplementation has been linked to hypocholesterolemic effects, ${ }^{16}$ hepatoprotective properties via hypolipidemic effects, ${ }^{17}$ blood pressure reductions in hypertensive patients, ${ }^{18,19}$ improved anemia and immunosenescence in senior citizens, ${ }^{20}$ and beneficial effects on blood lipid profiles in patients with metabolic dysfunction. ${ }^{21,22}$ The antioxidant and anti-inflammatory properties of $A$. platensi $^{23}$ and $A$. maxima ${ }^{24}$ have been shown to reduce tissue damage in rheumatoid arthritis in a rodent model. Therefore, due to the multiple documented health-supportive properties of blue-green algae, they are frequently used as an ingredient in dietary supplements.

A novel $A$. platensis-based aqueous cyanophyta extract $(\mathrm{ACE})$ is a natural extract that promotes anti-inflammatory and pain-relieving activity. The primary component of ACE is phycocyanin, which is known to act as an antioxidant and a selective COX-2 inhibitor ${ }^{13}$ with documented anti-inflammatory properties. ${ }^{8-10}$ In addition to phycocyanin, ACE contains antiinflammatory compounds different from phycocyanin yet with complementary anti-inflammatory properties. ${ }^{25}$ The purpose of the two clinical pilot studies reported here was to provide evidence for resolution of chronic pain during consumption of a phycocyanin-enriched extract of Arthrospira, known to possess synergistic anti-inflammatory effects.

\section{Materials and methods Consumable nutritional product and placebo}

An ACE derived from Arthrospira (subspecies platensis) was obtained from Cerule LLC, where the extract Cyactiv ${ }^{\mathrm{TM}}$ is produced by a proprietary process involving aqueous 
extraction technology. The raw material is A. platensis or A. maxima. The feedstock is prescreened to detect impurities and evaluate levels of phycocyanin in order to obtain the highest quality material available. The dried biomass is reconstituted, and the aqueous phase extracted, using mechanical separation, and dried. The product specifications are determined as defined by current Good Manufacturing Practice, with phycocyanin levels $>30 \%$ as determined using spectrophotometry at $620 \mathrm{~nm}$ absorbance. ${ }^{26}$ For Study I, the product was encapsulated in veggie caps with $500 \mathrm{mg} / \mathrm{capsule}$, for a daily dose of two capsules. For Study II, the product was encapsulated such that two capsules would contain $500 \mathrm{mg}$ ACE, $250 \mathrm{mg}$ ACE, or $0 \mathrm{mg}$ ACE (placebo). The placebo was made from rice starch and matched for color prior to encapsulation in veggie caps. Study participants were instructed to consume the daily dose with food.

\section{Human clinical pilot studies}

Two human clinical pilot studies were conducted on healthy human subjects with well-defined areas of moderate chronic joint pain affecting their activities of daily living. For both studies, 12 study participants were enrolled after obtaining written informed consent, as approved by Sky Lakes Medical Center Institutional Review Board (FWA 2603). Inclusion criteria were subjects of either sex, 45-75 years of age, eating a balanced Western diet, with $>6$ months of chronic pain in well-defined anatomical area(s) involving joints. Exclusion criteria were recent trauma that would affect pain scoring and recent changes in diet, supplements, or medication that could potentially affect joint health and pain scores. Daily consumption of over-the-counter pain medication and supplements that may be beneficial to joint health was not an exclusion criterion; however, subjects were instructed to maintain this consumption constant during the study.

\section{Study design I}

The initial study was conducted as an open-label single-arm 4-week study, where 12 subjects (Table 1) consumed $1 \mathrm{~g} \mathrm{ACE}$

Table I Demographics for Study I (open-label 4-week study consuming I g ACE daily)

\begin{tabular}{|c|c|c|c|}
\hline & Age, years & $\begin{array}{l}\text { BMI, kg/m² } \\
\text { (baseline) }\end{array}$ & $\begin{array}{l}\text { BMI, kg/m² } \\
\text { (4 weeks) }\end{array}$ \\
\hline $\begin{array}{l}\text { Average } \\
(\text { mean } \pm \text { SEM })\end{array}$ & $53.1 \pm 6.8$ & $31.4 \pm 8.5$ & $29.4 \pm 7.7^{*}$ \\
\hline Range & $40-62$ & |7.65-5|.| & | $7.4-48 . \mid$ \\
\hline \multicolumn{4}{|c|}{$\begin{array}{l}\text { Note: *The mild reduction in BMI seen over the } 4 \text {-week period was analyzed using } \\
\text { the "within-subject" paired } t \text {-test and was highly significant }(P<0.01) \text {. } \\
\text { Abbreviations: BMI, body mass index; ACE, aqueous cyanophyta extract; SEM, } \\
\text { standard error of the mean. }\end{array}$} \\
\hline
\end{tabular}

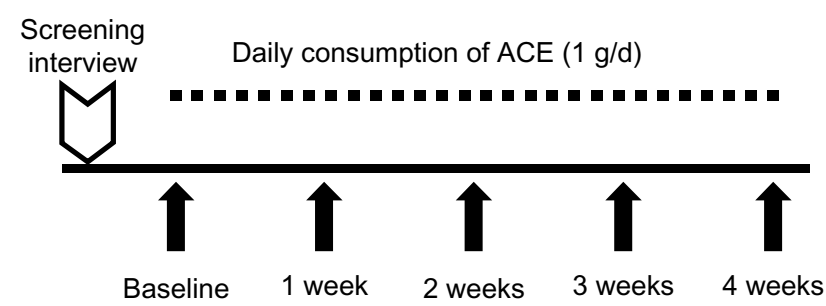

Figure I Study design for the open-label pilot study on the aqueous cyanophyta extract (ACE) from Arthrospira platensis.

Notes: One gram of ACE was consumed daily for 4 weeks. At baseline, each person's primary and secondary anatomical areas of chronic pain complaint were noted. Pain scores for these areas were collected at each subsequent visit, where each data collection visit is indicated by an arrow.

daily for the study duration (Figure 1). Questionnaire-based data collection happened on a weekly basis during visits to NIS Labs. Questionnaires included an activities of daily living questionnaire and pain scores for each person's primary and secondary area of chronic pain. The pain scores were determined from responses, using visual analog scales, to questions about pain both at rest and when physically active.

\section{Study design II}

The subsequent study was conducted as a single-blind crossover 5-week study, where 12 subjects (Table 2) consumed ACE or placebo daily for three 1-week periods, separated by 1 -week washout periods (Figure 2). Questionnaire-based data collection happened on a weekly basis during visits to NIS Labs, using the same questionnaires as in the initial open-label study.

\section{Statistical analysis}

Statistical significance of changes from baseline to later assessments was evaluated by "within-subject" analysis using the two-tailed, paired $t$-test. Statistical significance was indicated if $P<0.05$, and a high level of significance was indicated if $P<0.01$ and a very high level of significance if $P<0.001$.

\section{Results}

All study participants from both Study I and Study II completed study participation and reported good tolerability

Table 2 Demographics for Study II (placebo-controlled crossover dose study) ${ }^{\mathrm{a}}$

\begin{tabular}{lll}
\hline & Age, years & $\begin{array}{l}\text { BMI, kg/m } \\
\text { (baseline) }\end{array}$ \\
\hline $\begin{array}{l}\text { Average } \\
\text { (mean } \pm \text { SEM) }\end{array}$ & $58.3 \pm 8.2$ & $27.0 \pm 5.4$ \\
Range & $4 \mathrm{I}-72$ & $17.9-36.3$ \\
\hline
\end{tabular}

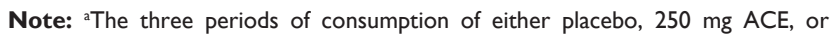
$500 \mathrm{mg}$ ACE were I week duration, separated by I week washout.

Abbreviations: BMI, body mass index; ACE, aqueous cyanophyta extract; SEM, standard error of the mean. 


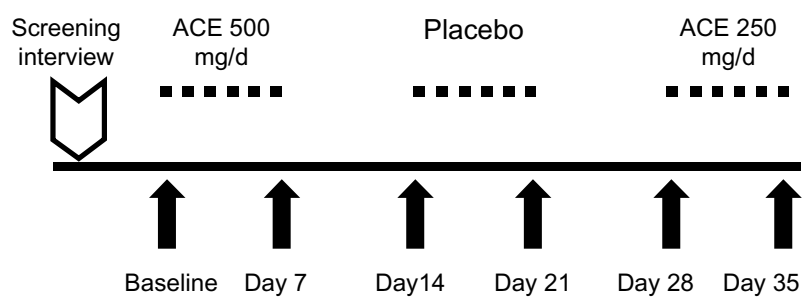

Figure 2 Study design for the single-blind, placebo-controlled dose study. Notes: Placebo ( $0 \mathrm{mg}$ product) as well as $250 \mathrm{mg}$ and $500 \mathrm{mg}$ aqueous cyanophyta extract (ACE) were each consumed for 7 days, by all study participants, separated by a 7-day washout period. The order in which each study participant consumed placebo versus $250 \mathrm{mg}$ and $500 \mathrm{mg} \mathrm{ACE}$ is as indicated on the diagram; however, the test products were of similar appearance and were blinded and unknown to the study participants. At baseline, each person's primary and secondary anatomical areas of chronic pain complaint were noted. Pain scores for these areas were collected at each subsequent visit, where each data collection visit is indicated by an arrow.

and absence of gastric upset. This was an important observation when consuming a known COX-2 inhibitor, phycocyanin, at a dose up to $300 \mathrm{mg} / \mathrm{d}(\sim 30 \%$ of the $1 \mathrm{~g} / \mathrm{d}$ dose of ACE).

The initial 4-week study also showed a very mild reduction in body weight as reflected by the body mass index (BMI) (Table 1). People with low BMI at study start showed only minimal change well within the range of normal daily variations, whereas people with higher BMI at study start showed a mild reduction in BMI such that the overall BMI changes in the study population at study end reached a high level of statistical significance when compared to BMI at study start $(P<0.01)$. The subsequent dose study did not monitor weight since the consumption periods were of only 1 week duration and used lower doses of product.

Significant changes in pain scores were seen in the initial 4-week study, during which time the study participants consumed $1 \mathrm{~g}$ ACE daily (Figure 3 ). The baseline pain score for each person's identified area of primary pain complaint at baseline was similar between pain at rest and pain during physical activity. In contrast, each person's secondary area of chronic pain was less aggravating as shown by lower score at rest but increased during physical activity. The pain reduction seen during consumption of ACE for 4 weeks showed rapid improvements for both the areas of primary and secondary pain. For the area of primary pain, the improvement reached a statistical trend $(P<0.1)$ at 1 week. The reduction continued and reached a high level of statistical significance $(P<0.01)$ at 2 weeks, after which the pain scores for the areas of primary pain plateaued. In contrast, the area of secondary pain during physical activity continued to show improvement for the entire 4 weeks, reaching a high level of statistical significance after 1 week $(P<0.01)$ and remained highly significant for the remainder of the study.
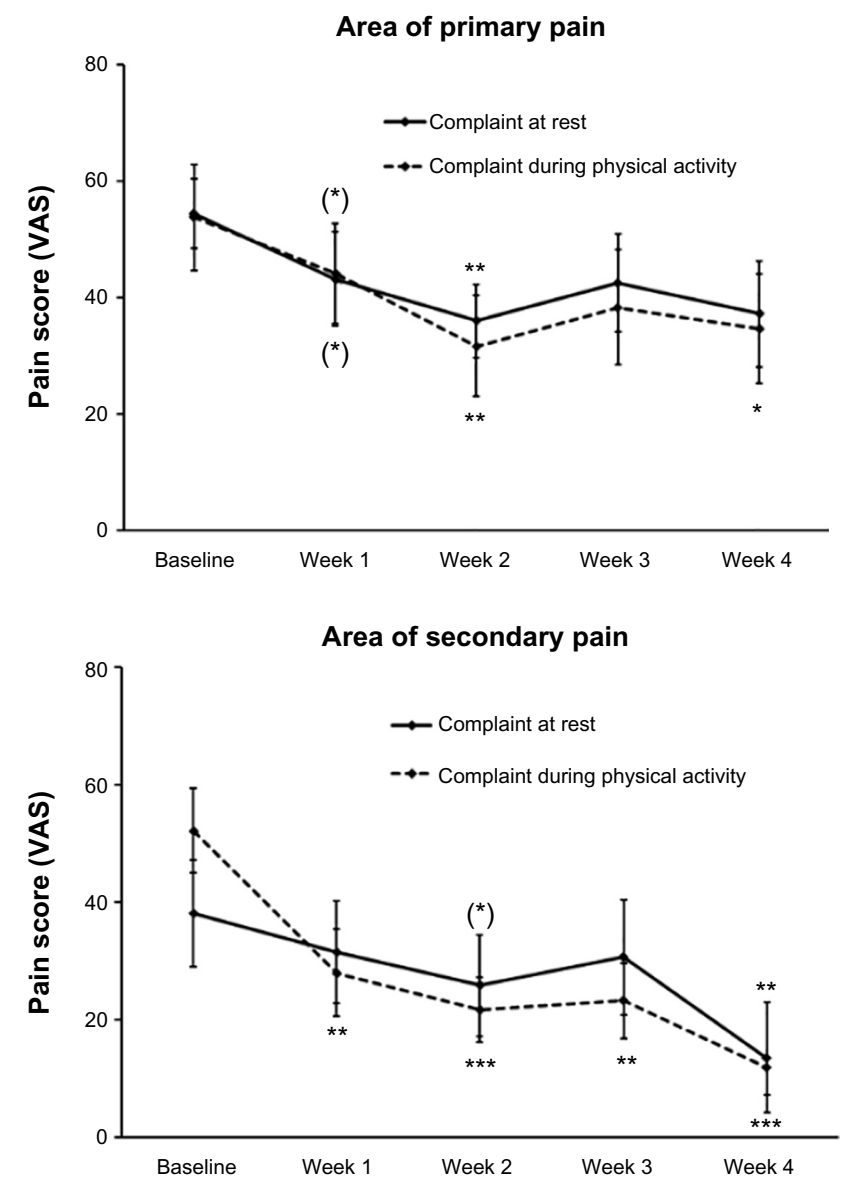

Figure 3 Data on pain scores collected by visual analog scales (VAS) during Study I.

Notes: Data are shown for the primary (top) and secondary (bottom) pain complaint areas when at rest (solid lines) and when physically active (dashed lines). The average \pm the standard error of the mean for the 12 study participants is shown for baseline and the four following weekly visits. Levels of significance of data sets when compared to baseline data are indicated by asterisks: a trend of $P<0.1$ is indicated by (*), significance $P<0.05$ indicated by *, high statistical significance $P<0.01$ indicated by **, and a very high level of statistical significance $P<0.001$ indicated by ***.

For the primary pain complaint, the pain levels at rest and when physically active were comparable at study start, showed a reduction that became significant after 2 weeks consumption $(P<0.01)$, and then plateaued, likely due to increased physical activity. The pain associated with the secondary pain complaint was lower at rest and higher during physical activity at baseline and showed rapid reduction, reaching significance $(P<0.01)$ after 1 week of consumption. The average pain scores for the secondary pain complaint were reduced to very low levels after 4 weeks, reaching a very high level of significance $(P<0.001)$.

For the subsequent dose study, where each dose of ACE (0 mg, $250 \mathrm{mg}$, and $500 \mathrm{mg}$ ) was consumed for a 1-week duration separated by 1 -week washout periods, the rapid improvement in chronic pain of a person's secondary pain complaint area during physical activity seen in Study I was 

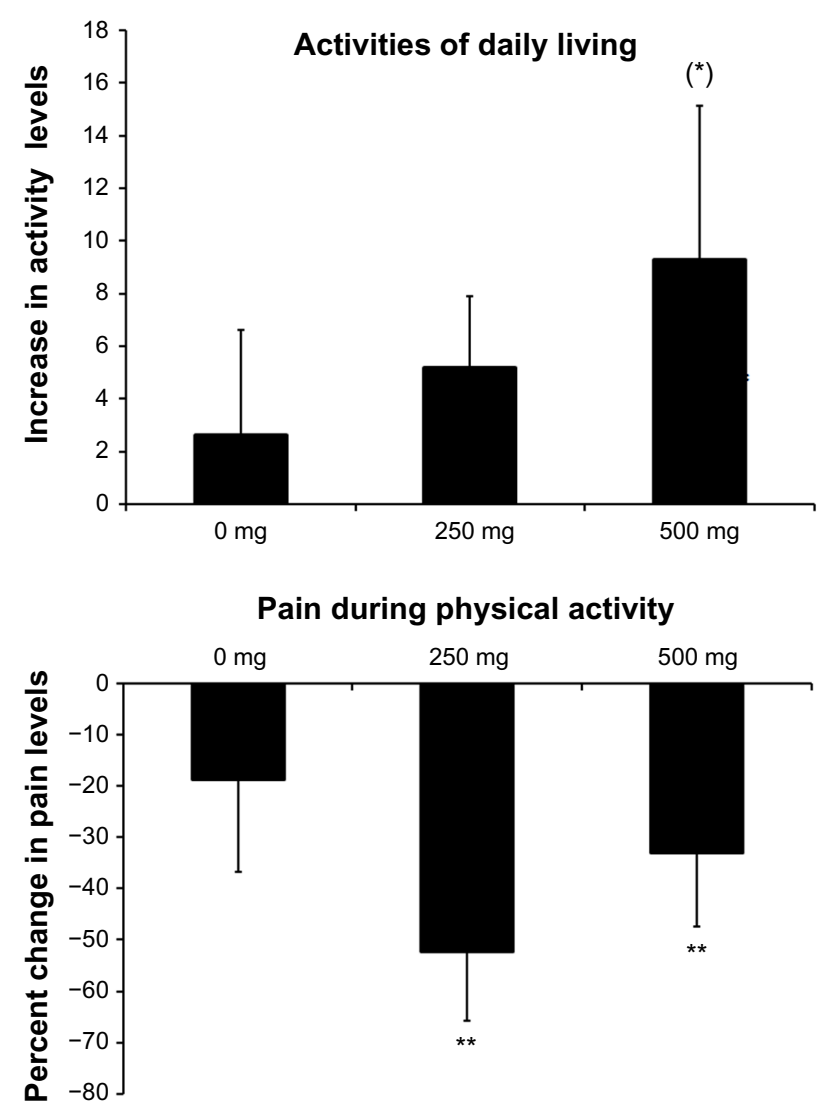

Figure 4 Data on activities of daily living questionnaire and pain level during physical activity were collected during Study II.

Notes: Data on activities of daily living (top) are shown as the average \pm the standard error of the mean for the changes recorded from baseline to the following visits, where either placebo $(0 \mathrm{mg})$ or ACE at $250 \mathrm{mg}$ or $500 \mathrm{mg}$ was consumed daily. Data on pain scores (bottom) are shown for those people with pain scores at baseline over $5 / 100$, thus excluding people with no pain during physical activities a

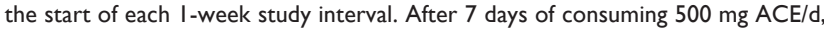
participants were increasingly active. At both ACE doses of $500 \mathrm{mg} / \mathrm{d}$ and $250 \mathrm{mg} / \mathrm{d}$ pain levels during physical activity were reduced. The combined data suggest that pain reduction allowed for an increased level of physical activity. Levels of significance of data sets when compared to baseline data are indicated by asterisks: a trend of $P<0.1$ is indicated by $(*)$ and a high level of statistical significance $P<0.01$ indicated by $* *$

Abbreviation: ACE, aqueous cyanophyta extract.

confirmed (Figure 4). Data on pain scores were analyzed for those people with pain scores at baseline above $5 / 100$, thus excluding people with no pain during physical activities at the start of each 1-week study interval. The reduced pain after 1-week consumption of both the $500 \mathrm{mg}$ and the $250 \mathrm{mg}$ doses was highly significant when compared to consumption of placebo $(P<0.01)$.

\section{Discussion}

The anti-inflammatory effects of crude Arthrospira products, as well as phycocyanin-enriched extracts, are well documented. ${ }^{8,9,23-25,27-29}$ However, to the best of our knowledge, this study is the first to establish an association with consumption of an aqueous Arthrospira extract enriched in phycocyanin and other aqueous compounds to pain relief in a human population. The overall speed and magnitude of resolution of some aspects of chronic pain suggests an underlying effect on oxidative stress and inflammation. Several parallel mechanisms of antioxidant and anti-inflammatory activity of ACE have shown that the bioactivities of the extract are more complex than what can be accounted for by the content of phycocyanin alone. The findings that ACE contains compounds capable of inhibiting the lipoxygenase inflammatory enzyme, ${ }^{23}$ in conjunction with its content of the COX-2 inhibitor phycocyanin, suggest that the rapid effects seen reflect, at least in part, a synergistic effect of inhibition of several inflammatory enzymes.

A complex relationship between reduction of pain scores and increased physical activity is a known factor in studies of relief of chronic pain. ${ }^{3}$ The scores for activities of daily living for this study population showed a dose-dependent relationship, with people reporting improved ability to perform daily activities during the week when they were consuming a $500 \mathrm{mg}$ dose of ACE.

The observation that people with a higher BMI at study start showed weight reduction during the 4-week consumption of ACE suggests that previously reported effects of Arthrospira-based nutritional products on metabolism ${ }^{22}$ are at least in part due to aqueous compounds. In light of the known associations between obesity and inflammation, ${ }^{30}$ a future study of longer duration is warranted and would monitor weight, body fat percentage, and metabolic hormones, as well as fasting glucose and insulin.

\section{Conclusion}

In conclusion, the data presented here show rapid effects on the resolution of chronic pain in people consuming ACE, accompanied by increased ability to perform daily activities. The documented effects of ACE on other aspects of inflammation, including inhibition of free radical formation by inflammatory cells ${ }^{23}$ suggest a multifaceted mechanism underlying the clinical observations. Future work is warranted, including a double-blind, randomized, placebo-controlled study on chronic joint-related pain, as well as evaluation of effects on inflammatory and metabolic biomarkers.

\section{Disclosure}

GSJ, KFB, SGC, and VLA are employees of NIS Labs. JG and AE are employed by Cerule LLC, the sponsor of this study. The authors report no other conflicts of interest in this work. 


\section{References}

1. Bingham B, Ajit SK, Blake DR, Samad TA. The molecular basis of pain and its clinical implications in rheumatology. Nat Clin Pract Rheumatol. 2009;5(1):28-37.

2. Woolf CJ, Doubell TP. The pathophysiology of chronic pain- increased sensitivity to low threshold Aß-fibre inputs. Curr Opin Neurobiol. 1994;4(4):525-534.

3. Benson KF, Ager DM, Landes B, Aruoma OI, Jensen GS. Improvement of joint range of motion (ROM) and reduction of chronic pain after consumption of an ergothioneine-containing nutritional supplement. Prev Med. 2012;54:S83-S89.

4. Kidd BL, Urban LA. Mechanisms of inflammatory pain. Br J Anaesth. 2001;87:3-11.

5. Coussens LM, Werb Z. Inflammation and cancer. Nature. 2002; 420:860-867.

6. Zhang JZ, An J. Cytokines, inflammation and pain. Anesthesiol Clin. 2007;45(2):27-37.

7. Maroon JC, Bost JW, Maroon A. Natural anti-inflammatory agents for pain relief. Surg Neurol Int. 2010;1:80.

8. Romay C, Armesto J, Remirez D, González R, Ledon N, García I. Antioxidant and anti-inflammatory properties of C-phycocyanin from blue-green algae. Inflamm Res. 1998;47(1):36-41.

9. Romay C, Ledón N, González R. Further studies on anti-inflammatory activity of phycocyanin in some animal models of inflammation. Inflamm Res. 1998;47(8):334-338.

10. Romay C, Ledón N, González R. Effects of phycocyanin extract on prostaglandin E2 levels in mouse ear inflammation test. Arzneimittelforschung. 2000;50(12):1106-1109.

11. Kalafati M, Jamurtas AZ, Nikolaidis MG, et al. Ergogenic and antioxidant effects of spirulina supplementation in humans. Med Sci Sports Exerc. 2010;42(1):142-151.

12. Ku CS, Pham TX, Park Y, et al. Edible blue-green algae reduce the production of pro-inflammatory cytokines by inhibiting NF- $\kappa \mathrm{B}$ pathway in macrophages and splenocytes. Biochim Biophys Acta. 2013;1830(4):2981-2988.

13. Reddy C, Vadiraja B, Bhat V, et al. Selective inhibition of cyclooxygenase-2 by C-Phycocyanin, a biliprotein from Spirulina platensis. Biochem Biophys Res Commun. 2000;277:599-603.

14. Romay C, González R, Ledón N, Remirez D, Rimbau V. C-phycocyanin: a biliprotein with antioxidant, anti-inflammatory and neuroprotective effects. Curr Protein Pept Sci. 2003;4(3):207-216.

15. Hur SJ, Kang SH, Jung HS, et al. Review of natural products actions on cytokines in inflammatory bowel disease. Nutr Res. 2012;32(11):801-816.

16. Samuels R, Mani UV, Iyer UM, Nayak US. Hypocholesterolemic effects of Spirulina in patients with hyperlipidemic nephrotic syndrome. J Med Food. 2002;5(2):91-96.

17. Torres-Duran PV, Ferreira-Hermosillo A, Juarez-Oropeza MA. Antihyperlipemic and antihypertensive effects of Spirulina maxima in an open sample of Mexican population: a preliminary report. Lipids Health Dis. 2007;6:33.
18. Juárez-Oropeza MA, Mascher D, Torres-Durán PV, Farias JM, Paredes-Carbajal MC. Effects of dietary Spirulina on vascular reactivity. J Med Food. 2009;12(1):15-20.

19. Lu J, Ren DF, Xue YL, Sawano Y, Miyakawa T, Tanokura M. Isolation of an antihypertensive peptide from alcalase digest of Spirulina platensis. J Agric Food Chem. 2010;58(12):7166-7171.

20. Selmi C, Leung PS, Fischer L, et al. The effects of Spirulina on anemia and immune function in senior citizens. Cell Mol Immunol. 2011;8(3):248-254.

21. Lee EH, Park JE, Choi YG, Huh KB, Kim WY. A randomized study to establish the effects of spirulina in type 2 diabetes mellitus patients. Nutr Res Pract. 2008;2(4):295-300.

22. Fujimoto M, Tsuneyama K, Fujimoto T, Selmi C, Gershwin ME, Shimada Y. Spirulina improves non-alcoholic steatohepatitis, visceral fat macrophage aggregation, and serum leptin in a mouse model of metabolic syndrome. Dig Liver Dis. 2012;44(9):767-774.

23. Ali EA, Barakat BM, Hassan R. Antioxidant and angiostatic effect of Spirulina platensis suspension in complete Freund's adjuvant-induced arthritis in rats. PLoS One. 2015;10(4):e0121523.

24. Gutiérrez-Rebolledo GA, Galar-Martínez M, García-Rodríguez RV, Chamorro-Cevallos GA, Hernández-Reyes AG, Martínez-Galero E. Antioxidant Effect of Spirulina (Arthrospira) maxima on chronic inflammation induced by Freund's complete adjuvant in rats. $\mathrm{J} \mathrm{Med}$ Food. 2015;18(8):865-871.

25. Jensen GS, Attridge VL, Beaman JL, Guthrie J, Ehmann A, Benson KF. Antioxidant and anti-inflammatory properties of an aqueous cyanophyta extract derived from Arthrospira platensis: contribution to bioactivities by the non-phycocyanin aqueous fraction. J Med Food. 2015;18(5):535-541.

26. Notice to US Food and Drug Administration that the use of CyaninPlus is Generally Recognized as Safe. Filed March 2012; GRAS Notice 424; Date of Closure December 6, 2012.

27. Remirez D, Gonzalez A, Merino N, et al. Effect of phycocyanin in zymosan-induced arthritis in mice-phycocyanin as an antiarthritic compound. Drug Dev Res. 1999;48(2):70-75.

28. Bhat VB, Madyastha KM. C-Phycocyanin: a potent peroxyl radical scavenger in vivo and in vitro. Biochem Biophys Res Commun. 2000; 225:20-25.

29. Reddy MC, Subhashini J, Mahipal SV, et al. C-Phycocyanin, a selective cyclooxygenase-2 inhibitor, induces apoptosis in lipopolysaccharidestimulated RAW 264.7 macrophages. Biochem Biophys Res Commun. 2003;304:385-392.

30. Johnson AR, Milner JJ, Makowski L. The inflammation highway: metabolism accelerates inflammatory traffic in obesity. Immunol Rev. 2012;249(1):218-238.
Nutrition and Dietary Supplements

\section{Publish your work in this journal}

Nutrition and Dietary Supplements is an international, peer-reviewed, open access journal focusing on research into nutritional requirements in health and disease, impact on metabolism and the identification and optimal use of dietary strategies and supplements necessary for normal growth and development. The journal welcomes papers covering

\section{Dovepress}

original research, basic science, clinical \& epidemiological studies, reviews and evaluations, guidelines, expert opinion and commentary, case reports and extended reports. The manuscript management system is completely online and includes a very quick and fair peer-review system, which is all easy to use. 